\title{
Perioperative management of patients with undergoing durable mechanical circulatory support
}

\author{
Nikolai Hulde, Andreas Koster, Vera von Dossow \\ Institute of Anesthesiology, Heart and Diabetes Centre North Rhine Westphalia, Ruhr-University Bochum, Bad Oeynhausen, Germany \\ Contributions: (I) Conception and design: All authors; (II) Administrative support: None; (III) Provision of study materials or patients: None; (IV) \\ Collection and assembly of data: None; (V) Data analysis and interpretation: None; (VI) Manuscript writing: All authors; (VII) Final approval of \\ manuscript: All authors. \\ Correspondence to: Vera von Dossow, MD. Director of the Institute of Anesthesiology, Heart and Diabetes Centre Bad Oeynhausen, Ruhr-University \\ Bochum, Georgstrasse 11, 32454 Bad Oeynhausen, Germany. Email: vvondossow@hdz-nrw.de.
}

\begin{abstract}
Durable mechanical circulatory support (MCS) devices revolutionized the treatment options for patients with end-stage heart failure (HF). Implantation of durable mechanical support has become an integral treatment modality in end-stage HF patients and it is associated with improved quality of life and survival. There is no doubt that this needs an interdisciplinary and interprofessional approach of cardiac surgeons, cardiologists, cardiac anesthesiologists, perfusionists, intensivists, psychologists, assist device coordinators as well as physiotherapists and intensive care. Implantation of durable MCS is a challenging procedure for the anesthesiologist due to the patient's characteristics and comorbid diseases. It demands comprehensive training, high vigilance and quick response during the acute hemodynamic changes occurring during the surgery. Preoperative risk stratification is of major importance to guide perioperative medical treatment strategies. Most of these patients have several comorbidities and multiple medications. Therefore, to anticipate postoperative end-organ dysfunction such as cognitive dysfunction, pulmonary or renal failure, an interdisciplinary approach is necessary to optimize patient's prior surgery. Transthoracic and transesophageal echocardiography (TTE and TEE), both play an invaluable role in diagnosing the cause and guiding the management in different unstable clinical situations. Especially prevention of postoperative right HF with subsequent necessity of temporary MCS is important as it is associated with higher mortality. The aim of this review is to provide an overview about the current concepts of perioperative management for durable MCS. A multimodal standard operating procedure supports early recovery after surgery and intensive care stay. Standardized perioperative care helps to ensure optimal medical treatment. This review focusses on several major skills of perioperative management of these high-risk surgical patients.
\end{abstract}

Keywords: Perioperative anesthesia management; preoperative evaluation; early recovery after LVAD implantation

Submitted Mar 22, 2020. Accepted for publication May 13, 2020.

doi: $10.21037 / \mathrm{atm}-20-2527$

View this article at: http://dx.doi.org/10.21037/atm-20-2527

\section{Introduction}

Heart failure (HF) has become one of the most frequently cardiovascular diseases worldwide (1-3). Despite optimal medical management, an increasing number of patients progress to end-stage HF with poor quality of life, recurrent hospitalizations and increased mortality (4-6). Even if cardiac transplantation remains the gold standard of care for these patients with excellent outcomes, it is severely limited due to the availability of donor hearts.

Recently the European Association of Cardio-Thoracic Surgery (EACTS) Expert Consensus group published their expert consensus statement on long-term (LT) mechanical circulatory support (MCS) for patients with advanced 
HF (7). According to these recommendations LT-MCS implantation is indicated in patients with New York Heart Association (NYHA) functional class III $\mathrm{b}$ and IV and an ejection fraction $\leq 25 \%$ and at least one of the criteria inotropic support, temporary MCS support, peak oxygen consumption $\mathrm{VO}_{2}<12 \mathrm{~mL} / \mathrm{kg}$ min. Several LEVELS are defined by the Interagency Registry for Mechanical Assisted Circulatory Support (INTERMACS) LEVEL 1-6 (7-9). LT-MCS at INTERMACS LEVEL 1 is associated with poorer outcome then LEVELS 2-4 $(8,10)$. In these patients bridging with temporary MCS (i.e., VA ECMO, Impella) is recommended (LEVEL B) $(10,11)$. Patients who are designated INTERMACS Level 3 and 4 may be best served with a planned LT implantable continuous-flow left ventricular (LV) assist device (cf-LVAD). Current studies confirmed improved quality of life and survival of nearly $80 \%$ at 1 -year and $70 \%$ at 2 -year from INTERMACS registry data (12-14) (Table 1).

\section{Durable MCS classification}

Durable MCS devices can be categorized depending by the pump flow type (pulsatile or continuous), engineering design (first, second, third generation), left, right or biventricular support. First-generation MCS are large pulsatile flow devices $(15,16)$. Implantation of these devices was associated with an increased risk of infections and bleeding (15). During the last two decades technical evolution started the era of continuous flow (CF) type devices, the second and third generation MCS. The secondgeneration devices employ contact-bearing design and axial pattern of blood flow (HeartMate II) whereas thirdgeneration devices employ non-contact bearing design (using magnetic levitation) with centrifugal blood flow $(12,17)$. Nowadays the most implanted third generation devices are HeartWare Ventricular Assist Device $\left(\mathrm{HVAD}^{\circledR}\right)$ and HeartMate 3. They consist of a centrifugal CF pump, both are small and allow intrapericardial implantation with minimal-invasive surgical technique (18).

The first randomized study of durable MCS was published in 2001 (19). The REMATCH study ("Randomized Evaluation of Mechanical Assistance for the treatment of Congestive heart Failure") was a cornerstone in the era of durable MCS and showed improved life quality and $48 \%$ risk reduction of death for patients compared to patients with optimal medical therapy (19). In accordance to these results the ROADMAP study, an observational study in 200 ambulatory HF patients, confirmed improved life quality and functional status after Heartmate II implantation compared to optimal medical treatment (20).

Several previous studies investigated comparison thirdgeneration versus second-generation devices with respect to outcome and complications: $\mathrm{HVAD}^{\circledR}$ was approved 2012 by the Food and Drug Administration (FDA). The ADVANCE trial, revealed $86 \%$ of 1 -year survival after implantation (21). In addition, the ENDURANCE Trial compared HVAD ${ }^{\circledR}$ and Heartmate II (13). The results showed noninferiority after 2 years (55\% versus $57 \%$ ) with respect to survival free of cerebral infarction or pump replacement for the HVAD $^{\circledast}$ compared to HeartMate II. Despite a lower rate of technical defects, $\mathrm{HVAD}^{\circledast}$ was associated with an increased rate of cerebral infarction (13). The MOMENTUM 3 study compared HeartMate 3 versus HeartMate II. HeartMate 3 was associated with a better 1- and 2-year survival of $84 \%$ and $75 \%$ and no pump thrombosis (14). Recently, Schramm et al. revealed comparable 1 -year survival rate $(75.5 \%$ versus $76.0 \%$ ) for $\mathrm{HVAD}^{\circledR}$ and HeartMate 3 (22). In addition, the rate for cerebrovascular incidents did not differ between both systems.

For patients with severe biventricular failure, total artificial heart (TAH) offers a final alternative for lomg-term support. The first TAH Jarvik 7 was implanted successfully by William de Vries $1986(17,23,24)$. It consisted of two pneumatic replacement pumps and transcutaneous air power tubes and was connected to a computer-unit. On the basis of this initial technology, CardioWest and now SynCard TAH (SynCARDIA Systems, Inc. Houston, TX, USA) has been developed and is currently the only approved TAH (FDA approval since 2004) (25).

Despite technological improvements, bleeding, thromboembolism, cerebral stroke and bleeding, pump thrombosis, driveline infections and device failure still remain important issues and affect the long-term outcome of patients on durable MCS (17). It is recommended that such devices should only be implanted and managed at centers with a multidisciplinary team approach and trained physicians and nursing staff (26).

\section{Implication for preoperative risk stratification}

A multidisciplinary team is an integral approach assessing patient's suitability for durable MCS which is summarized in the consensus statement of EACTS on "long-term (LT)" MCS 2019 (7). These consensus recommendations include clear statements on indications and contraindications of LTMCS. The EACTS expert consensus statement summarizes 
Table 1 INTERMACS-classification (adapted to 5)

\begin{tabular}{|c|c|c|c|}
\hline Profile & Clinical situation & Device & 1-year survival \\
\hline 1 & "Crash and burn" & & \\
\hline \multirow[t]{2}{*}{2} & Progressive decline, inotropic therapy, NYHA IV & Temporary MCS & $63.1 \% \pm 3.1 \%$ \\
\hline & "Slinding on inotropes" & & \\
\hline 3 & “dependent stability" & & \\
\hline \multirow[t]{2}{*}{4} & Resting symptoms, NYHA IV & LT-MCS LVAD & $78.7 \% \pm 3.0 \%$ \\
\hline & "frequent flyer" & & \\
\hline 5 & Exertion intolerant, NYHA IV & LT-MCS/LVAD & $93.0 \% \pm 3.9 \%$ \\
\hline 6 & "walking wounded" & & \\
\hline \multirow[t]{2}{*}{7} & Advanced NYHA class III & LVAD discussion & n.a. \\
\hline & "Placeholder" & & \\
\hline
\end{tabular}

Table 1 is adapted from Ponikowski P, Voors AA, Anker SD, et al. 2016 ESC Guidelines for the Diagnosis and Treatment of Acute and Chronic Heart Failure. Rev Esp Cardiol (Engl Ed) 2016;69:1167 (5). MCS, mechanical circulatory support; LT, long-term; NYHA, New York Heart Association; LVAD, left ventricular assist device.

important aspect of patient's characteristics in the evaluation for LT-MCS. This include comorbidities, frailty, renal and hepatic dysfunction, the role of diabetes mellitus as well as hemostatic deficiencies. Cognitive dysfunction, dementia, active drug consumption, severe inflammation(bacterial/ fungal) as well as irreversible organ dysfunction (renal, hepatic) are important contraindications in the EACTS expert consensus statement (7). They recommend a fullsite patient evaluation and preoperative organ function optimization. In this context, a multidisciplinary approach with the cardiac surgeon, cardiologist, anesthesiologists as well as intensivist is mandatory to improve outcome after LT-MCS implantation.

\section{Preoperative diagnostic tools}

\section{Cognitive and functional assessment}

Anesthetic pre-assessment should include an evaluation of fitness for durable MCS surgery/anesthesia, an appraisal of existing comorbidities and identification for opportunities to improve perioperative care. A cognitive and functional assessment is available to define vulnerability and degree of frailty of these patients (27-29). It includes the clock drawing test and or mini mental testing, hand grip strength, Tinetti Test, Time up and go as well as 6 min walk. Patients with end-stage $\mathrm{HF}$ are less able to sustain increased oxygen consumption which is associated with cardiac surgery and subsequent systemic inflammatory response. In addition, age and frailty increase the vulnerability of these patients (30-32). Therefore, a structured and safe pre-habilitation program might increase aerobic capacity. Cardiac prehabilitation includes a multicomponent program (aerobic exercise training, respiratory muscle training, weight control, nutritional optimization, improvement of sleep patterns, psychosocial assessment and education) and has been demonstrated to reduce postoperative complications (30-32). From the pathophysiological point of view prerehabilitation aerobic exercise seems to have the favorable effects, but it still remains to be determined in patients undergoing durable MCS.

\section{TTE and TEE}

The 2015 American Society of Echocardiography (ASE) 
recommend both TTE and TEE to evaluate patients pre- intra- and (33-35) preoperative echocardiographic assessment with TTE/TEE provides valuable information about following skills:

(I) the status of $\mathrm{LV}$ and right ventricular (RV) cardiac systolic function [evaluation of the degree of RV dysfunction/biventricular assist device (BIVAD)];

(II) the degree of pulmonary arterial hypertension $(\mathrm{PAH})$

(III) the presence of valvulopathies:

(i) aortic regurgitation: risk of poor systemic perfusion;

(ii) aortic stenosis: prevention of $L V$ ejection;

(iii) mitral stenosis prevents LV flow resulting in poor LVADS flow;

(iv) tricuspid regurgitation (TR) is associated with RV dysfunction.

(IV) intracardiac thrombus increased risk of systemic embolism from thrombus LAA/LV;

(V) the size of the left ventricle: difficult inflow cannula placement;

(VI) persistent foramen ovale;

(VII) other congenital defects and pericardial effusion.

Prior durable MCS implantation, critical transthoracic echocardiographic evaluation of the RV function is obligatory due to the fact that postoperative RV failure increases postoperative mortality (36). In these cases, temporary RVAD support is necessary until RV function is recompensated. Nowadays, there is neither a standardized protocol nor consensus recommendations for the preoperative medical optimization prior LVAD implantation to prevent right HF. Most centers use their own protocols. In addition, no controlled randomized studies are available and outcome data are still lacking. The EACTS consensus statement noticed that levosimendan administration prior LVAD implantation might be indicated (7).

Pre and Post RV systolic function is the main concern. Apart from eyeball RV function (good, moderate, poor) there are several objective methods used such as fractional area change (FAC) and Tricuspid Annular Plane Systolic Excursion (TAPSE). FAC is $32-60 \%$ (RV FAC (\%) = EDA - EDS)/EDA; and a normal TAPSE value is $>16 \mathrm{~mm}$. A higher right over LV end diastolic - area ratio (RVEDA/ LVEDA) was associated with increased 60-day mortality in LVAD patients $(0.7+0.09$ versus $0.62+0.11, \mathrm{P}<0.01)(37,38)$. A recently published meta-analysis of 36 observational studies including 4,428 patients after LVAD implantation, found that the pooled LV diameter was smaller in patients who developed RHF, as opposed to the RV, which was larger in patients with RV failure (39). In accordance to these findings Otten and colleagues observed that a smaller LV diameter was associated with a worse outcome (40). Another important echocardiographic parameter is TR which is associated with $\mathrm{RV}$ volume overload resulting in RV dilatation (41). The incidence of moderate and severe TR in patients with end-stage HF undergoing LVAD implantation is relatively high, and is reported to be in the range of $12-57 \%$ (42-44). There are different reports regarding the clinical value of TR after LVAD implantation. Milano et al. analyzed 382 patients who received LVADs (HeartWare) (42). They found a significantly increased risk of RHF or death up to 30 days after implantation in patients with moderate or severe TR before implantation [odds ratio (OR) 2.9 (1.8-4.8; $\mathrm{P}<0.0001)$ ]. Goldraich et al. reported a similar high incidence of severe TR of $70 \%$ in patients with tricuspid annulus dilatation $\left(>23 \mathrm{~mm} / \mathrm{m}^{2}\right)(43)$. Both, tricuspid annulus dilatation and severe TR were independent predictors of RHF [OR 6.00 (1.36-26.37; $\mathrm{P}<0.02)$ and $\mathrm{OR} 4.62(0.88-24.18 ; \mathrm{P}<0.07)]$. In accordance to these results recent reports from the INTERMACS Registry of patients with LVAD demonstrated that moderate and severe TR at the time of LVAD implantation were associated with higher mortality (44). However, the value of preoperative echocardiographic examination in predicting RV HF in patients after the implantation of LVAD remains to be still determined due to the lack of randomized trials and different definitions of right HF. Previous studies examined small and heterogenous patient cohorts, most of them were analyzed retrospectively. In addition, the indications for LVAD implantation, the type of device implanted as well as the definition of RHF varied among the centers (45).

\section{Noninvasive tests to predict $R H$ failure}

There are several noninvasive tests to assess RV function (46). Cardiac MRI is useful to quantify RV function and morphology in $\mathrm{PAH}$ patients. The first-pass radionuclide angiography with radiolabeled RBC is the gold standard for calculating RV function. Gated blood pool SPECT can be an alternative as it separates the cardiac chambers well and provides accurate assessment of the RV function with high reproducibility, which is particularly useful for monitoring treatment. However, more research is needed to compare and validate these modalities in evaluating $\mathrm{RV}$ function in a perioperative setting (46). 


\section{Assessment of renal function}

The incidence of acute renal insufficiency (AKI) after cardiac surgery is reported to be $22-36 \%$ (47). Severe AKI requiring renal replacement therapy is associated with a mortality of $50-80 \%$ (48). Preoperative renal assessment should include clinical risk stratification and measurement of serum creatinine concentration to estimate basal glomerular filtration rate (GFR). Modifiable risk factors should be optimized prior surgery (diabetes, hypertension, nephrotoxic drugs). The major impact of renal dysfunction on durable MCS outcomes warrants close collaboration with a nephrologist in the evaluation of a reduced GFR in order to optimize all reversible causes. Therapeutic drug monitoring is mandatory to reduce pharmacological interactions and negative side effects, especially in case of polypharmacy (48).

\section{Preoperative fasting}

Intake of clear fluids until 2 hours before surgery does not increase gastric content, reduce gastric $\mathrm{pH}$ or increase complication rates. In case of absence of delayed gastric emptying, the intake of fluids two hours before induction of anesthesia is recommended (49). Oral carbohydrate loading reduces postoperative insulin resistance and improves patients comfort (50).

\section{Preanesthetic medication}

Routine administration of benzodiazepines to decrease preoperative anxiety should be avoided (49). Previous studies showed that long-acting benzodiazepines are associated with an increased time on the ventilator and delayed extubation resulting in an increased rate of postoperative delirium (51). In addition, Maurice-Szamburski and colleagues demonstrated that self-reported experience after surgery was not improved with the benzodiazepine lormetazepam as premedication (52).

However, short-acting benzodiazepines can be used in extremely anxious patients with caution (51). Alternatively, Melatonin administered 1-2 hours before surgery reduces preoperative anxiety (53). Non-pharmacological strategies such as music interventions or relaxation techniques as well as hypnosis might offer an alternative approach to reduce preoperative anxiety (54).

\section{Anesthesia induction and maintenance}

In HF patient's hemodynamics depend on high catecholamine levels in the circulation. Anesthetics can block the sympathetic system and can cause blood pressure lability resulting in hypotension and requiring vasopressors. Due to the reduced ejection fraction, drugs are distributed slower into the circulation resulting in delayed anesthetic effects. The anesthesia induction is among the most critical periods of durable MCS with an increased risk of cardiovascular collapse. The principle concern is right heart dysfunction due to several trigger factors such as $\mathrm{PAH}$, decreased sympathetic tone, reduced systemic venous return and a depressed myocardium. In addition, beginning of positive pressure ventilation after intubation can increase right heart ventricular afterload. Several high-risk patients require hemodynamic preoptimization with appropriate vasoactive support prior induction to preserve systemic vascular resistance, $\mathrm{RV}$ myocardial contractility as well as avoidance of increase of pulmonary resistance.

The anesthetic management should focus on short to middle-acting narcotics and opioids that permit early spontaneous breathing and early extubation after surgery. Agents such as etomidate, midazolam, ketamine are mandatory for induction of anesthesia. Etomidate has neither negative inotropic and chronotropic effect nor does it cause myocardial depression. Midazolam induction was found to maintain cardiovascular stability in end-stage HF patients. Opioids are useful to prevent sympathetic response to laryngoscope. Long-acting opioids should be avoided and middle-short -acting opioids such as sufentanil with optimal pharmacokinetic profile should be used in combination with ketamine. Ketamine does not increase the pulmonary vascular resistance in patients with severe pulmonary hypertension and has bronchodilating effects. In contrast propofol should be used with caution due to its negative inotropic and chronotropic effects.

Induction of anesthesia requires high clinical vigilance and slow administration of the agent of choice with continuous evaluation of physiological responses. All alterations require immediate treatment as they can be detrimental for the patient. The major goal of induction of anesthesia is to maintain hemodynamic stability. Surgeon and perfusionist should be immediately available during induction and should be prepared to urgently perform sternotomy, cannulation and cardio-circulatory assistance.

\section{Intraoperative TEE}

An initial intraoperative TEE exam assesses the patient's cardiac status and pulmonary pressures at the time of 
the procedure and serves as a reference to intraoperative and postoperative findings. Intraoperative TEE allows a dynamic assessment after durable MCS implantation. The following assessment is mandatory after LVAD implantation:

(I) LV should be monitored with caution with respect to an adequate filling (Prevention of suction event with collapse of the LV cavity);

(II) LVAD inflow cannula should be placed ideally in a central position in the LV cavity. No interference to the mitral valve apparatus is mandatory;

(III) Laminar flow through mitral valve should be mandatory into the LV and LVAD inflow cannula

(IV) Paradoxical septum shift should be excluded;

(V) adequate de-airing of $\mathrm{LV}$ is mandatory;

(VI) Recognition of early RV dysfunction: RV systolic function, TR severity, and interventricular septum position as well as systemic venous return (distention of the right ventricle);

(VII) Aortic regurgitation should be excluded;

(VIII) postoperative complications such as tamponade/ hemothorax should be excluded.

\section{Recognition of early $R V$ failure}

The definition of RV is defined as unplanned insertion of an RVAD or the use of an intravenous inotrope for longer than 14 days $(55,56)$. Numerous studies have been published regarding echocardiographic parameters and its impact in predicting RV failure $(42-45,55,56)$. Evaluation of $\mathrm{RV}$ in the operating room (OR) includes: $\mathrm{RV}$ chamber size, visual estimation of RV contractility, presence of new or severe TR jet, TAPSE, Tricuspid annular systolic velocity, interventricular septal position and contractility in systole and diastole as well as pulmonary artery (PA) systolic pressure by assessing TR jet peak velocity (doppler echocardiography) adding it to central venous pressure. Evaluation of the TV is essential as worsening TR may indicate a dilated $\mathrm{RV}$ from $\mathrm{RV}$ failure, $\mathrm{PAH}$ or fluid overload.

Pulmonary regurgitation $(\mathrm{PR})$ is often seen in patients with PAH along with PA dilatation. Color Flow Doppler (CFD) imaging will detect a diastolic jet in the right ventricular outflow tract (RVOT) and pathological PR has a wider, holodiastolic jet. Regurgitation velocities are measured by continuous wave Doppler (CWD) to calculate mean pulmonary artery pressure (MPAP). Right heart catheterization as gold standard for diagnosis of PA pressures is mandatory and confirm the level of the PAP.

In summary, these echocardiographic parameters play an important role in the decision making process regarding anesthesia induction and utilization of inotropic and vasopressor support at the beginning of the case.

\section{Intraoperative monitoring}

Perioperative monitoring is to enable early detection of changes in specific physiologic parameters. The progression of these parameters might result in adverse consequences. Basic cardiorespiratory and metabolic monitoring (ECG, NIBP, invasive blood pressure monitoring, end-tidal $\mathrm{CO}_{2}$, temperature) should be used in all patients. Preinduction invasive arterial pressure monitoring is obligatory as hemodynamic instability can occur around anesthesia induction. In case of veno-arterial (VA)-ECMO prior durable MCS implantation, the right upper limb is the preferred site for continuous $\mathrm{SPO}_{2}$ monitoring.

Even if numerous studies have failed to demonstrate any outcome benefit from PA catheter use in various patient cohorts, PA catheterization facilitates continuous monitoring of the dynamic changes in central-venous pressure (CVP) and PA pressures as well as the monitoring of treatment interventions (positive inotropes, inhalational pulmonary vasodilator therapy with milrinone, iloprost and NO) (57). More sophisticated PA catheters allow estimation of RV end-diastolic and end-systolic volume together with $\mathrm{RV}$ ejection fraction $(58,59)$.

\section{Neurological monitoring}

In all patients undergoing durable MCS depth of anesthesia should be measured. A recent Cochrane review of randomized trials of non-ICU found BIS-guided depth of anesthesia was associated with lower rates of postoperative delirium (60). In accordance to these findings the European Association of Anesthesiology recommends monitoring of depths of anesthesia to reduce the incidence of postoperative delirium (49). The use of near infrared spectroscopy (NIRS) in cardiac surgery is quite common. However, there is only low evidence linking NIRS-detected regional cerebral oxygen desaturation during cardiac surgery with neurological complications after surgery (61). There has been no conclusive consensus between anesthesiologists and perfusionists regarding appropriate criteria for the use of cerebral oximetry (62). In patients undergoing LVAD implantation higher $\mathrm{rSO}_{2}$ values at baseline are associated 
with lower 30-day mortality with an odds ratio of 0.94 and $95 \%$ confidence interval $(0.888,0.995)$ for every $1 \%$ increase of $\mathrm{rSO}_{2}(63)$.

\section{Intraoperative management of PAH and right heart dysfunction}

$\mathrm{PAH}$ is defined as a mean pulmonary arterial pressure $>25 \mathrm{~mm}$, measured by right heart catheterization $(64,65)$. As chronic end-stage HF progresses, the left and right ventricle adapt by enlarging their volumes. But firstly, the right ventricle first compensates with remodelling of the extracellular matrix and concentric hypertrophy. In case of increased RV afterload due to $\mathrm{PAH}$, the ventricle becomes dilated with consecutive impaired function resulting in RV failure and shock. Patients with severe PAH and RV dysfunction are at high risk to collapse during induction of anesthesia which is due to nearly all anesthetics (negative inotropic effects), anesthesia-induced sympatholysis with arterial and venous dilation. This results in systemic hypotension, decreased RV preload and reduced coronary artery perfusion pressure. The first sign is leftward shifting of the septum in the TEE. Therefore, pre-emptive VA ECMO might be indicated in patients with preexisting severe PAH (66).

In addition, pre-existing PAH might aggravate in the perioperative period due to several trigger factors that should be avoided: These are hypoxia, hypercapnia, acidosis and hypothermia, all known to increase pulmonary vascular resistance (PVR) and to induce pulmonary vasoconstriction. Lung protective ventilation with low inspiratory pressures and low tidal volumes is mandatory and protects the right ventricle.

\section{Therapeutically implications}

The major goals in the management and prevention of RV dysfunction include pulmonary vasodilation, optimization of the RV, systemic vasoconstriction and maintenance of sinus rhythm.

\section{Pulmonary vasodilation}

Pulmonary vasodilation can be achieved using three different therapeutical approaches: $\mathrm{NO}$ as direct inhibitor of phosphodiesterase-type $\mathrm{V}(\mathrm{PDE}-\mathrm{V})$ and activation of soluble guanylate cyclase (sGC) resulting in smooth muscle relaxation and PVR reduction. Despite a wide use of inhalational NO in durable MCS patients, there is still limited evidence-based data available (lack of randomized -controlled trials). Potapov et al. failed to demonstrate a beneficial effect in LVAD patients with prophylactic 40 ppm NO application (67). However, in several patients NO application has been demonstrated to be a potent vasodilator improving RV contractility $(68,69)$.

An alternative approach is the endogenous prostacyclin pathway. Prostaglandin I2 increases intracellular cyclic adenosine monophosphate. (cAMP) levels resulting in vasodilatation and consecutive positive inotropic effects in acute PH conditions (70). Inhaled PGI2 (epoprostenol) results in selective pulmonary vasodilation and has been studied in various patient collectives (71). Currently a prospective randomized trial compares the effect of iNO and iPGI2 in patients undergoing LVAD implantation, heart and lung transplantation (NCT 030381052, ClinicalTrials. gov).

Inhaled iloprost is a carbacyclin analog of PGI2 exerting its vasodilatory effects of 60-90 minutes. Previous studies reported superior effect compared to iNO and inhalational sildenafil in cardiothoracic surgery $(72,73)$. Iloprost was more effective in reducing RV afterload (74). In summary, vasodilators should be administered via inhalation as "selective pulmonary vasodilators". The intravenous application should be avoided due to an increased risk of RV failure, systemic hypotension and shock.

\section{Optimization of $R V$ performance}

Inotropes are classified in sympathomimetic agents (epinephrine, dobutamine, norepinephrine), phosphodiesterase-type III inhibitors (milrinone, enoximone) and calcium sensitizers (levosimendan). Prophylactic use of inotropes is not recommended. Dobutamine is the standard inotropic agent in cardiac surgery with $\beta 1$-adrenergic effects. Previous studies suggested that in patients with low ejection fraction $(<40 \%)$ the effect of dobutamine might be reduced compared to patients with normal contractility (75). That means, in severe HF patients the $\beta$-receptor density was lower, nearly $50 \%$. Infusion of dobutamine was associated with a reduced contractility response in these patients (75). In contrast, selective phosphodiesterase-type III inhibitors (enoximone, milrinone) increase intracellular levels of cyclic adenosine monophosphate and consecutively increase contractility. This positive effect has to be regard with caution, as it is often accompanied by vasodilating effects and systemic 
hypotension. As several patients already receive milrinone preoperatively as long-term treatment (INTERMAC III), they have an increased risk of severe systemic hypotension after anesthesia induction. Despite its beneficial inotropic effects on cardiac contractility, it remains unclear whether phosphodiesterase-type III inhibitor milrinone have a beneficial or detrimental effect on mortality (76-78). For enoximone there are still no data available.

The positive inotropic effect of levosimendan results from increasing the sensitivity of cardiac troponin $\mathrm{C}$ to calcium (called calcium sensitizer), and the opening of mitochondrial KTP channels of smooth muscle cells. In contrast to all other inotropic substances, myocardial oxygen consumption is not increased with levosimendan. This is an important fact as the early postoperative period is associated with an increased oxygen consumption and systemic inflammatory response syndrome. Therefore, preoperative administration of levosimendan seems reasonable as preconditioning in patients with pre-existent RV dysfunction. Due to its pharmacokinetic profile, the maximal inotropic effect of levosimendan would be mostly effective given one day before surgery (79). In a patients with cardiogenic shock, the infusion of levosimendan significantly improved RV contractility (80). In addition, levosimendan has anti-inflammatory and renal protective effects, which could be beneficial in these patients known to have a proinflammatory status. However, there are limited data available and its effect on mortality has to be still be determined yet.

\section{Systemic vasoconstriction}

Systemic vasoconstriction results in increased coronary artery perfusion. Adrenaline and noradrenaline are sympathomimetic vasopressors causing systemic and pulmonary vasoconstriction as well as RV contractility. Vasopressin is known to cause systemic vasoconstriction via stimulating G-protein-coupled V1 receptors of vascular myocytes (81). In humans, vasopressin seems not to affect the pulmonary vascular tone. Therefore, in patients with $\mathrm{PH}$ it seems the drug of primary choice treating arterial hypotension (77-79). In patients with $\mathrm{PH}$ and $\mathrm{RV}$ dysfunction systemic hypotension should be strictly avoided. Noradrenalin and vasopressin are the primary choice to prevent systemic hypotension in patients undergoing durable MCS.

\section{Conclusions}

Durable MCS are implanted for a great variety of indications at different clinical situations. Management of these patients requires professional education and expertise as these patients are critically ill with various comorbidities. Therefore, the anesthesiologist carrying for these critically ill patients should be vigilant about the pathophysiology of HF, the operative procedure and the implanted devices. A preoperative assessment of all organ functions is mandatory. Different treatment options and anticipation of complications should be discussed in a multidisciplinary team approach. RV failure is one of the major complications and should be prevented whenever possible. Further randomized trials are necessary to define preoperative assessment tools and to integrate preemptive pre-rehabilitation programs with respect to enhanced recovery after cardiac surgery recommendations. This might improve outcome of these critically ill patients.

\section{Acknowledgments}

Funding: None.

\section{Footnote}

Provenance and Peer Review: This article was commissioned by the Guest Editor (Kamen Valchanov) for the series "Perioperative Management of Patients with undergoing Mechanical Circulatory Support" published in Annals of Translational Medicine. The article was sent for external peer review organized by the Guest Editor and the editorial office.

Conflicts of Interest: All authors have completed the ICMJE uniform disclosure form (available at http://dx.doi. org/10.21037/atm-20-2527). The series "Perioperative Management of Patients with undergoing Mechanical Circulatory Support" was commissioned by the editorial office without any funding or sponsorship. The authors have no other conflicts of interest to declare.

Ethical statement: The authors are accountable for all aspects of the work in ensuring that questions related to the accuracy or integrity of any part of the work are appropriately investigated and resolved.

Open Access Statement: This is an Open Access article distributed in accordance with the Creative Commons Attribution-NonCommercial-NoDerivs 4.0 International License (CC BY-NC-ND 4.0), which permits the non- 
commercial replication and distribution of the article with the strict proviso that no changes or edits are made and the original work is properly cited (including links to both the formal publication through the relevant DOI and the license). See: https://creativecommons.org/licenses/by-nc-nd/4.0/.

\section{References}

1. Miller RJH, Teuteberg JJ, Hunt SA. Innovations in Ventricular Assist Devices for End-Stage Heart Failure. Annu Rev Med 2019;70:33-44.

2. Roger VL. Epidemiology of heart failure. Circ Res 2013;113:646-59.

3. Braunwald $\mathrm{E}$. The war against heart failure: the Lancet lecture. Lancet 2015;385:812-24.

4. Yancy CW, Jessup M, Bozkurt B, et al. 2017 ACC/ AHA/HFSA Focused Update of the 2013 ACCF/AHA Guideline for the Management of Heart Failure: A Report of the American College of Cardiology/American Heart Association Task Force on Clinical Practice Guidelines and the Heart Failure Society of America. J Card Fail 2017;23:628-51.

5. Ponikowski P, Voors AA, Anker SD, et al. 2016 ESC Guidelines for the Diagnosis and Treatment of Acute and Chronic Heart Failure. Rev Esp Cardiol (Engl Ed) 2016;69:1167.

6. Boruah P, Saqib N, Barooah J, et al. Left Ventricular Assist Device: What the Internist Needs to Know. A Review of the Literature. Cureus 2019;11:e4399.

7. Potapov EV, Antonides C, Crespo-Leiro MG, et al. 2019 EACTS Expert Consensus on long-term mechanical circulatory support. Eur J Cardiothorac Surg 2019;56:230-70.

8. Stevenson LW, Pagani FD, Young JB, et al. INTERMACS profiles of advanced heart failure: the current picture. J Heart Lung Transplant 2009;28:535-41.

9. Boyle AJ, Ascheim DD, Russo MJ, et al. Clinical outcomes for continuous-flow left ventricular assist device patients stratified by pre-operative INTERMACS classification. J Heart Lung Transplant 2011;30:402-7.

10. Jorde UP, Kushwaha SS, Tatooles AJ, et al. Results of the destination therapy post-food and drug administration approval study with a continuous flow left ventricular assist device: a prospective study using the INTERMACS registry (Interagency Registry for Mechanically Assisted Circulatory Support). J Am Coll Cardiol 2014;63:1751-7.

11. Riebandt J, Haberl T, Mahr S, et al. Preoperative patient optimization using extracorporeal life support improves outcomes of INTERMACS Level I patients receiving a permanent ventricular assist device. Eur J Cardiothorac Surg 2014;46:486-92; discussion 492.

12. Acharya D, Loyaga-Rendon R, Morgan CJ, et al. INTERMACS Analysis of Stroke During Support With Continuous-Flow Left Ventricular Assist Devices: Risk Factors and Outcomes. JACC Heart Fail 2017;5:703-11.

13. Rogers JG, Pagani FD, Tatooles AJ, et al. Intrapericardial Left Ventricular Assist Device for Advanced Heart Failure. N Engl J Med 2017;376:451-60.

14. Mehra MR, Salerno C, Cleveland JC, et al. Healthcare Resource Use and Cost Implications in the MOMENTUM 3 Long-Term Outcome Study. Circulation 2018;138:1923-34.

15. Meng ML, Spellman J. Anesthetic management of the patient with a ventricular assist device. Best Pract Res Clin Anaesthesiol 2017;31:215-26.

16. Lahpor JR. State of the art: implantable ventricular assist devices. Curr Opin Organ Transplant 2009;14:554-9.

17. Kirklin JK, Naftel DC, Pagani FD, et al. Seventh INTERMACS annual report: 15,000 patients and counting. J Heart Lung Transplant 2015;34:1495-504.

18. Rojas SV, Avsar M, Hanke JS, et al. Minimally invasive ventricular assist device surgery. Artif Organs 2015;39:473-9.

19. Rose EA, Gelijns AC, Moskowitz AJ, et al. Long-term use of a left ventricular assist device for end-stage heart failure. N Engl J Med 2001;345:1435-43.

20. Estep JD, Starling RC, Horstmanshof DA, et al. Risk Assessment and Comparative Effectiveness of Left Ventricular Assist Device and Medical Management in Ambulatory Heart Failure Patients: Results From the ROADMAP Study. J Am Coll Cardiol 2015;66:1747-61.

21. Aaronson KD, Slaughter MS, Miller LW, et al. Use of an intrapericardial, continuous-flow, centrifugal pump in patients awaiting heart transplantation. Circulation 2012;125:3191-200.

22. Schramm R, Zittermann A, Morshuis M, et al. Comparing short-term outcome after implantation of the HeartWare(R) HVAD(R) and the Abbott(R) HeartMate 3(R). ESC Heart Fail 2020. [Epub ahead of print].

23. Griffith BP, Hardesty RL, Kormos RL, et al. Temporary use of the Jarvik-7 total artificial heart before transplantation. N Engl J Med 1987;316:130-4.

24. Jarvik RK, DeVries WC, Semb BK, et al. Surgical positioning of the Jarvik-7 artificial heart. J Heart Transplant 1986;5:184-95.

25. Copeland JG, Smith RG, Arabia FA, et al. Cardiac 
replacement with a total artificial heart as a bridge to transplantation. N Engl J Med 2004;351:859-67.

26. Lambrinou E, Kalogirou F, Lamnisos D, et al. Effectiveness of heart failure management programmes with nurse-led discharge planning in reducing readmissions: a systematic review and meta-analysis. Int J Nurs Stud 2012;49:610-24.

27. Dasgupta M, Rolfson DB, Stolee P, et al. Frailty is associated with postoperative complications in older adults with medical problems. Arch Gerontol Geriatr 2009;48:78-83.

28. Makary MA, Segev DL, Pronovost PJ, et al. Frailty as a predictor of surgical outcomes in older patients. J Am Coll Surg 2010;210:901-8.

29. Waite I, Deshpande R, Baghai M, et al. Home-based preoperative rehabilitation (prehab) to improve physical function and reduce hospital length of stay for frail patients undergoing coronary artery bypass graft and valve surgery. J Cardiothorac Surg 2017;12:91.

30. Kamarajah SK, Boyle C, Bundred JR, et al. Critical appraisal of gastric conduit ischaemic conditioning (GIC) prior to oesophagectomy: A systematic review and metaanalysis. Int J Surg 2020;77:77-82.

31. Yau DKW, Wong MKH, Wong WT, et al. PREhabilitation for improving QUality of recovery after ELective cardiac surgery (PREQUEL) study: protocol of a randomised controlled trial. BMJ Open 2019;9:e027974.

32. Bottiger BA, Nicoara A, Snyder LD, et al. Frailty in the End-Stage Lung Disease or Heart Failure Patient: Implications for the Perioperative Transplant Clinician. J Cardiothorac Vasc Anesth 2019;33:1382-92.

33. Perrino AC Jr, Reeves ST. editors. A Practical Approach to Transesophageal Echocardiography. Philadelphia: LIPPINCOTT WILLIAMS \& WILKINS, 2013.

34. Hahn RT, Abraham T, Adams MS, et al. Guidelines for performing a comprehensive transesophageal echocardiographic examination: recommendations from the American Society of Echocardiography and the Society of Cardiovascular Anesthesiologists. J Am Soc Echocardiogr 2013;26:921-64.

35. Stainback RF, Estep JD, Agler DA, et al. Echocardiography in the Management of Patients with Left Ventricular Assist Devices: Recommendations from the American Society of Echocardiography. J Am Soc Echocardiogr 2015;28:853-909.

36. Patangi SO, George A, Pauli H, et al. Management issues during HeartWare left ventricular assist device implantation and the role of transesophageal echocardiography. Ann Card Anaesth 2013;16:259-67.

37. Kukucka M, Potapov E, Stepanenko A, et al. Acute impact of left ventricular unloading by left ventricular assist device on the right ventricle geometry and function: effect of nitric oxide inhalation. J Thorac Cardiovasc Surg 2011;141:1009-14.

38. Vivo RP, Cordero-Reyes AM, Qamar U, et al. Increased right-to-left ventricle diameter ratio is a strong predictor of right ventricular failure after left ventricular assist device. J Heart Lung Transplant 2013;32:792-9.

39. Bellavia D, Iacovoni A, Scardulla C, et al. Prediction of right ventricular failure after ventricular assist device implant: systematic review and meta-analysis of observational studies. Eur J Heart Fail 2017;19:926-46.

40. Otten A, Kurz S, Anwar S, et al. Prognostic value of 3-dimensional echocardiographical heart volume assessment in patients scheduled for left ventricular assist device implantation. Eur J Cardiothorac Surg 2018;54:169-75.

41. Kiernan MS, Grandin EW, Brinkley M Jr, et al. Early Right Ventricular Assist Device Use in Patients Undergoing Continuous-Flow Left Ventricular Assist Device Implantation: Incidence and Risk Factors From the Interagency Registry for Mechanically Assisted Circulatory Support. Circ Heart Fail 2017;10:e003863.

42. Milano C, Pagani FD, Slaughter MS, et al. Clinical outcomes after implantation of a centrifugal flow left ventricular assist device and concurrent cardiac valve procedures. Circulation 2014;130:S3-11.

43. Goldraich L, Kawajiri H, Foroutan F, et al. Tricuspid Valve Annular Dilation as a Predictor of Right Ventricular Failure After Implantation of a Left Ventricular Assist Device. J Card Surg 2016;31:110-6.

44. Song HK, Gelow JM, Mudd J, et al. Limited Utility of Tricuspid Valve Repair at the Time of Left Ventricular Assist Device Implantation. Ann Thorac Surg 2016;101:2168-74.

45. Paluszkiewicz L, Borgermann J. The value of echocardiographic examination in predicting right ventricular heart failure in patients after the implantation of continuous-flow left ventricular assist devices. Interact Cardiovasc Thorac Surg 2018;27:931-7.

46. Ramani G, Chen W, Patel S, et al. Noninvasive Assessment of Right Ventricular Function in Patients with Pulmonary Arterial Hypertension and Left Ventricular Assist Device. Curr Cardiol Rep 2019;21:82.

47. Hu J, Chen R, Liu S, et al. Global Incidence and Outcomes of Adult Patients With Acute Kidney Injury 
After Cardiac Surgery: A Systematic Review and MetaAnalysis. J Cardiothorac Vasc Anesth 2016;30:82-9.

48. Kidney Disease Improving global outcomes (KDIGO) Clinical Practice Guideline for Acute Kidney Injury. Kidney International Supplements 2012;2:1-138.

49. Aldecoa C, Bettelli G, Bilotta F, et al. European Society of Anaesthesiology evidence-based and consensus-based guideline on postoperative delirium. Eur J Anaesthesiol 2017;34:192-214.

50. Breuer JP, von Dossow V, von Heymann C, et al. Preoperative oral carbohydrate administration to ASA IIIIV patients undergoing elective cardiac surgery. Anesth Analg 2006;103:1099-108.

51. Batchelor TJP, Rasburn NJ, Abdelnour-Berchtold E, et al. Guidelines for enhanced recovery after lung surgery: recommendations of the Enhanced Recovery After Surgery (ERAS(R)) Society and the European Society of Thoracic Surgeons (ESTS). Eur J Cardiothorac Surg 2019;55:91-115.

52. Maurice-Szamburski A, Auquier P, Viarre-Oreal V, et al. Effect of sedative premedication on patient experience after general anesthesia: a randomized clinical trial. JAMA 2015;313:916-25.

53. Hansen MV, Halladin NL, Rosenberg J, et al. Melatonin for pre- and postoperative anxiety in adults. Cochrane Database Syst Rev 2015;2015:CD009861.

54. Bradt J, Dileo C, Shim M. Music interventions for preoperative anxiety. Cochrane Database Syst Rev 2013;(6):CD006908.

55. Riebandt J, Haberl T, Wiedemann D, et al. Extracorporeal membrane oxygenation support for right ventricular failure after left ventricular assist device implantation. Eur J Cardiothorac Surg 2018;53:590-5.

56. Potapov EV, Loforte A, Weng Y, et al. Experience with over 1000 implanted ventricular assist devices. J Card Surg 2008;23:185-94.

57. Sandham JD, Hull RD, Brant RF, et al. A randomized, controlled trial of the use of pulmonary-artery catheters in high-risk surgical patients. N Engl J Med 2003;348:5-14.

58. Richard C, Warszawski J, Anguel N, et al. Early use of the pulmonary artery catheter and outcomes in patients with shock and acute respiratory distress syndrome: a randomized controlled trial. JAMA 2003;290:2713-20.

59. Rajaram SS, Desai NK, Kalra A, et al. Pulmonary artery catheters for adult patients in intensive care. Cochrane Database Syst Rev 2013:CD003408.

60. Siddiqi N, Harrison JK, Clegg A, et al. Interventions for preventing delirium in hospitalised non-ICU patients.
Cochrane Database Syst Rev 2016;3:CD005563.

61. Zheng F, Sheinberg R, Yee MS, et al. Cerebral nearinfrared spectroscopy monitoring and neurologic outcomes in adult cardiac surgery patients: a systematic review. Anesth Analg 2013;116:663-76.

62. Zacharias DG, Lilly K, Shaw CL, et al. Survey of the clinical assessment and utility of near-infrared cerebral oximetry in cardiac surgery. J Cardiothorac Vasc Anesth 2014;28:308-16.

63. Ghosal S, Trivedi J, Chen J, et al. Regional Cerebral Oxygen Saturation Level Predicts 30-Day Mortality Rate After Left Ventricular Assist Device Surgery. J Cardiothorac Vasc Anesth 2018;32:1185-90.

64. Hoeper MM, Bogaard HJ, Condliffe R, et al. Definitions and diagnosis of pulmonary hypertension. J Am Coll Cardiol 2013;62:D42-50.

65. Galiè N, Humbert M, Vachiery JL, et al. 2015 ESC/ERS Guidelines for the diagnosis and treatment of pulmonary hypertension: The Joint Task Force for the Diagnosis and Treatment of Pulmonary Hypertension of the European Society of Cardiology (ESC) and the European Respiratory Society (ERS): Endorsed by: Association for European Paediatric and Congenital Cardiology (AEPC), International Society for Heart and Lung Transplantation (ISHLT). Eur Heart J 2016;37:67-119.

66. Biscotti M, Vail E, Cook KE, et al. Extracorporeal membrane oxygenation with subclavian artery cannulation in awake patients with pulmonary hypertension. ASAIO J 2014;60:748-50.

67. Potapov E, Meyer D, Swaminathan M, et al. Inhaled nitric oxide after left ventricular assist device implantation: a prospective, randomized, double-blind, multicenter, placebo-controlled trial. J Heart Lung Transplant 2011;30:870-8.

68. Benedetto M, Romano R, Baca G, et al. Inhaled nitric oxide in cardiac surgery: Evidence or tradition? Nitric Oxide 2015;49:67-79.

69. Deja M, Hommel M, Weber-Carstens S, et al. Evidencebased therapy of severe acute respiratory distress syndrome: an algorithm-guided approach. J Int Med Res 2008;36:211-21.

70. Zeineh NS, Bachman TN, El-Haddad H, et al. Effects of acute intravenous iloprost on right ventricular hemodynamics in rats with chronic pulmonary hypertension. Pulm Circ 2014;4:612-8.

71. McGinn K, Reichert M. A Comparison of Inhaled Nitric Oxide Versus Inhaled Epoprostenol for Acute Pulmonary Hypertension Following Cardiac Surgery. Ann 
Pharmacother 2016;50:22-6.

72. Hoeper MM, Olschewski H, Ghofrani HA, et al. A comparison of the acute hemodynamic effects of inhaled nitric oxide and aerosolized iloprost in primary pulmonary hypertension. German PPH study group. J Am Coll Cardiol 2000;35:176-82.

73. Leuchte HH, Schwaiblmair M, Baumgartner RA, et al. Hemodynamic response to sildenafil, nitric oxide, and iloprost in primary pulmonary hypertension. Chest 2004;125:580-6.

74. Winterhalter M, Simon A, Fischer S, et al. Comparison of inhaled iloprost and nitric oxide in patients with pulmonary hypertension during weaning from cardiopulmonary bypass in cardiac surgery: a prospective randomized trial. J Cardiothorac Vasc Anesth 2008;22:406-13.

75. Fowler MB, Laser JA, Hopkins GL, et al. Assessment of the beta-adrenergic receptor pathway in the intact failing human heart: progressive receptor down-regulation and subsensitivity to agonist response. Circulation 1986;74:1290-302.

76. Zangrillo A, Biondi-Zoccai G, Ponschab M, et al.

Cite this article as: Hulde N, Koster A, von Dossow V. Perioperative management of patients with undergoing durable mechanical circulatory support. Ann Transl Med 2020;8(13):830. doi: $10.21037 /$ atm-20-2527
Milrinone and mortality in adult cardiac surgery: a metaanalysis. J Cardiothorac Vasc Anesth 2012;26:70-7.

77. Greco T, Calabro MG, Covello RD, et al. A Bayesian network meta-analysis on the effect of inodilatory agents on mortality. Br J Anaesth 2015;114:746-56.

78. Nielsen DV, Torp-Pedersen C, Skals RK, et al. Intraoperative milrinone versus dobutamine in cardiac surgery patients: a retrospective cohort study on mortality. Crit Care 2018;22:51.

79. Toller W, Heringlake M, Guarracino F, et al. Preoperative and perioperative use of levosimendan in cardiac surgery: European expert opinion. Int J Cardiol 2015;184:323-36.

80. Russ MA, Prondzinsky R, Carter JM, et al. Right ventricular function in myocardial infarction complicated by cardiogenic shock: Improvement with levosimendan. Crit Care Med 2009;37:3017-23.

81. Tayama E, Ueda T, Shojima T, et al. Arginine vasopressin is an ideal drug after cardiac surgery for the management of low systemic vascular resistant hypotension concomitant with pulmonary hypertension. Interact Cardiovasc Thorac Surg 2007;6:715-9. 